\title{
New patterns of infecting organisms in late bleb-related endophthalmitis
}

The new paper on this subject by Waheed et al. ${ }^{1}$ presents a timely and useful review of experience with late bleb-related endophthalmitis over the last 10 years. All their patients with a clinical diagnosis of conjunctival injection, bleb purulence and intraocular inflammation (with or without cells in the vitreous cavity) underwent a vitreous aspirate for culture. This study has thus clearly identified the types of bacterial pathogens that can colonise the conjunctival or lid margin surface and then invade intraocular tissues. Such endophthalmitis developed after an average of 25 months (range 1-96 months) postoperatively. Anti-fibrotic agents had been used in 25 of 39 patients $(64 \%)$ suggesting a risk associated with their use, although the authors did not quote figures for those without infection. Bleb leaks were recognised in $65 \%$ of cases, and probably occurred in others as well, which can be considered the source or route of bacterial invasion.

Positive intraocular cultures have been identified in previous series, ${ }^{2-5}$ with a range of $67-97 \%$, which can be compared with $86 \%$ in Waheed et al.'s paper. In contrast, however, the microbial spectrum identified is similar to only one of the previous studies, ${ }^{3}$ when

staphylococci caused 56\% and streptococci 19\% of cases in Waheed et al.'s study.

Of the 25 staphylococcal cases, 13 were due to Staphylococcus aureus and 12 to coagulasenegative staphylococci (CNS). S. aureus is a much more virulent bacterium than CNS, and in common with other types of endophthalmitis ${ }^{6}$ final visual acuity (VA) was considerably reduced compared with CNS infection. S. aureus colonises approximately $10 \%$ of lid margins but this can increase to $70 \%$ in atopic subjects and $25 \%$ in those with chronic blepharitis. Unfortunately, neither of these risk factors was assessed by Waheed et al., but both should be considered for possible preventive strategies. This could include long-term intermittent therapy with a topical antistaphylococcal antibiotic such as Fucithalmic (fucidin in gel), but this should not be used continuously or fucidin-resistant staphylococci will quickly emerge. CNS colonise all lid margins so there is no preventive strategy to pursue here except to avoid producing blebs that leak. Interestingly, however, 7 of $12(58 \%)$ cases of CNS infection were not associated with any apparent leak from the bleb compared with only 2 of $13(15 \%)$ cases due to S. aureus infection.

Of the 8 streptococcal cases documented by Waheed et al. there was no apparent difference in VA outcome for S. viridans compared with S. pneumoniae and S. pyogenes infection. This is unusual and surprising. The latter virulent streptococci usually cause an acute purulent and devastating endophthalmitis often with all loss of VA. ${ }^{6,7}$

Types of antibiotics used for therapy of the endophthalmitis were not considered by Waheed et al. nor did they include antibiotic sensitivity results from the laboratory, which would have been useful data. Waheed et al. did use the intravitreal route for antibiotic therapy, which is to be encouraged. ${ }^{7}$

When intraocular inflammation is developing in a chronic condition, rather than acute circumstances, a trial of therapy with oral azithromycin or clarithromycin - new azilide derivatives of erythromycin - may be of benefit. These new erythromycin derivatives are well absorbed by the oral route and penetrate particularly well into cells including polymorphonuclear cells (extracellular to intracellular ratio of $1: 224$ ) with $30 \%$ of the serum level expected to penetrate into the eye. ${ }^{7}$ They are also bactericidal at low concentrations for staphylococci, streptococci and $H$. influenzae - covering 35 of 42 (83\%) positive cultures in Waheed et al.'s series - and are variously effective against the other seven positive cultures identified. Clarithromycin has been found effective recently in a case of saccular endophthalmitis following cataract surgery and intraocular lens placement, ${ }^{8}$ in a situation in which staphylococci have been found to be 'parasitising' macrophages to induce chronic inflammation. ${ }^{9}$ Acute infection, however, must still be treated with intravitreal antibiotics. ${ }^{7}$

Prevention of late-onset, bleb-associated endophthalmitis in the future will be linked with development of anti-fibrotic agents that do
Dr David V. Seal, MD, 23 Charleton Place Islington London N1 8AQ 
not predispose to leakage from the bleb opening up a route for bacterial invasion. In the meantime, careful use of topical antibiotics in selected patients may help prevent cases due to $S$. aureus but will be unlikely to reduce conjunctival and lid colonisation with the various other bacteria including the CNS identified by Waheed et al.

\section{References}

1. Waheed S, Ritterband DC, Greenfield DS, Liemann JM, Seedor JA, Ritch R. New patterns of infecting organisms in late blebrelated endophthalmitis: a ten-year review. Eye 1998;12:910-5.

2. Mandelbaum S, Forster RK, Gelende H, Culbertson W. Late onset endophthalmitis associated with filtering blebs. Ophthalmology 1985;92:964-72.
3. Phillips WB, Wong TP, Berger RL. Late-onset endophthalmitis associated with filtering blebs. Ophthalmic Surg 1994;25:88-91.

4. Greenfield DS, Suner IJ, Miller MP. Endophthalmitis after filtering surgery with mitomycin C. Arch Ophthalmol 1996;114:943-9.

5. Kangas TA, Greenfield DS, Flynn HW. Delayed-onset endophthalmitis associated with conjunctival filtering blebs. Ophthalmology 1997;104:746-52.

6. Verbraeken H. Treatment of post-operative endophthalmitis Ophthalmologica 1995;209:165-71.

7. Seal DV, Bron AJ, Hay J. Ocular infection: investigation and treatment in practice. London: Martin Dunitz, 1998:1-275.

8. Warheker PT, Gupta SR, Mansfield DC, Seal DV. Successful treatment of saccular endophthalmitis with clarithromycin. Eye 1998;12:1017-9.

9. Warheker PT, Gupta SR, Mansfield DC, Seal DV, Lee WR. Post-operative saccular endophthalmitis caused by macrophage-associated staphylococci. Eye 1998;12:1019-21. 